\title{
Postoperative atrial fibrillation in patients undergoing coronary artery bypass grafting or cardiac valve surgery: intraoperative use of landiolol
}

\author{
Kazuhiro Nakanishi", Shinhiro Takeda, Chol Kim, Shusuke Kohda and Atsuhiro Sakamoto
}

\begin{abstract}
Background: Landiolol hydrochloride is a new $\beta$-adrenergic blocker with a pharmacological profile that suggests it can be administered safely to patients who have sinus tachycardia or tachyarrhythmia and who require heart rate reduction. This study aimed to investigate whether intraoperative administration of landiolol could reduce the incidence of atrial fibrillation (AF) after cardiac surgery.

Methods: Of the 200 consecutive patients whose records could be retrieved between October 2006 and September 2007, we retrospectively reviewed a total of 105 patients who met the inclusion criteria: no previous permanent/persistent AF, no permanent pacemaker, no renal insufficiency requiring dialysis, and no reactive airway disease, etc. Landiolol infusion was started after surgery had commenced, at an infusion rate of $1 \mu \mathrm{g} / \mathrm{kg} / \mathrm{min}$, titrated upward in 3-5 $\mathrm{\mu g} / \mathrm{kg} / \mathrm{min}$ increments. The patients were divided into 2 groups: those who received intraoperative $\beta$-blocker therapy with landiolol (landiolol group) and those who did not receive any $\beta$-blockers during surgery (control group). An unpaired $t$ test and Fisher's exact test were used to compare between-group differences in mean values and categorical data, respectively.

Results: Seventeen of the 105 patients (16.2\%) developed postoperative atrial fibrillation: 5/57 (8.8\%) in the landiolol group and 12/48 (25\%) in the control group. There was a significant difference between the two groups $(\mathrm{P}=0.03)$. The incidence of $\mathrm{AF}$ after valve surgery and off-pump coronary artery bypass grafting was lower in the landiolol group, although the difference between the groups was not statistically significant.

Conclusions: Our retrospective review demonstrated a marked reduction of postoperative AF in those who received landiolol intraoperatively. A prospective study of intraoperative landiolol for preventing postoperative atrial fibrillation is warranted.
\end{abstract}

Keywords: $\beta$-blocker, Landiolol, Postoperative atrial fibrillation, Cardiac surgery

\section{Background}

Atrial fibrillation (AF) is the most common arrhythmic complication after cardiac surgery, with an incidence of approximately $20 \%$ to $50 \%$ that has not changed despite improvements in anesthesia, surgical techniques, and drug therapy [1]. This is probably due to co-morbidities including older age, left ventricular dysfunction, chronic pulmonary disease, and renal insufficiency, which are also

\footnotetext{
* Correspondence: n-kazu@nms.ac.jp

Department of Anesthesiology, Nippon Medical School, 1-1-5 Sendagi, Bunkyo-ku, Tokyo 113-8603, Japan
}

contributors to postoperative AF [2,3]. AF has also been associated with a complicated postoperative course, increased incidence of stroke, increased intensive care unit and total hospital stays, and increased health care costs [2-5].

Prophylaxis with preoperative $\beta$-blocker therapy [1], preoperative amiodarone [6], and postoperative atrial pacing [7] has had varying degrees of clinical impact, but recent reviews reflect a growing consensus in favor of prophylactic administration of $\beta$-blockers $[8,9]$. The latest American College of Chest Physicians guidelines for 
the prevention and management of postoperative AF after cardiac surgery recommended that strong consideration should be given to the prophylactic administration of $\beta$-blockers as means of lowering the incidence of new-onset post-cardiac surgery AF (strength of recommendation, A; evidence grade, fair, net benefit, substantial) [8]. However, the role of intraoperative $\beta$-adrenergic blockers in preventing postoperative AF is still unknown.

Landiolol hydrochloride is a new $\beta$-adrenergic blocker synthesized by Ono Pharmaceutical Co. (Osaka, Japan) that resembles esmolol, but has a greater $\beta 1$-selectivity and a shorter elimination half-life [10,11]. Landiolol is metabolized very quickly by serum pseudocholinesterase and carboxyesterase in the liver to an inactive metabolite with a half-life of $4 \mathrm{~min}$ in healthy human subjects [10]. Renal and hepatic clearance do not contribute to the pharmacokinetics at clinical concentrations, which makes landiolol titratable, and the onset and offset of action are rapid [10]. Landiolol has a higher $\beta 1$-selectivity than any currently available $\beta$-blocker, but has neither intrinsic sympathomimetic activity nor significant membranestabilizing activity [10-13]. Landiolol may suppress ventricular and supraventricular arrhythmias [13-15]. The superior pharmacological profile of landiolol may allow for safer use in patients in the acute phase of severe heart disease and in other clinical settings [15]. Therefore, we undertook a retrospective, single-institution study to determine if intraoperative administration of landiolol reduces the incidence of AF after cardiac surgery.

\section{Methods}

We reviewed the records of 200 consecutive patients who underwent elective coronary and/or valve surgery at our institution between October 2006 and September 2007, and who were managed by senior anesthesiologists and cardiologists. (This should signify that the subjects were patients who underwent cardiac surgery performed by surgeons from the same cardiac surgery department, in succession, during a one-year period between October 2006 and September 2007). Patients with previous permanent/persistent AF, a permanent pacemaker, renal insufficiency requiring dialysis and reactive airway diseases were excluded from the analysis, as were those who received amiodarone as initial therapy for AF before initiation of $\beta$-blocker therapy. Patients with prior AF were not excluded if they presented for surgery in sinus rhythm without amiodarone therapy. A total of 105 patients met these criteria for review. Infusions of landiolol were started after the beginning of surgery, at the discretion of the attending senior anesthesiologist. Infusion of landiolol was initiated at an infusion rate of $1 \mu \mathrm{g} / \mathrm{kg} / \mathrm{min}$ and titrated upward in 3-5 $\mu \mathrm{g} / \mathrm{kg} / \mathrm{min}$ increments based on heart rate $(\mathrm{HR})$ and blood pressure responses. The patients were divided into 2 groups: those who received intraoperative $\beta$-blocker therapy with landiolol (landiolol group) and those who did not receive any $\beta$-blockers during surgery (control group). After surgery, each patient was admitted to the intensive care unit (ICU) and was subsequently transferred to the ward. The patients were continuously monitored during the first $72 \mathrm{~h}$ after surgery, using bedside monitors that were alarm-triggered. Thereafter all patients were monitored routinely with an alarm-triggered three lead telemetry system until the morning of postoperative day 7. Postoperative $\beta$-blocker therapy (landiolol, carvedilol or atenolol) was used at the discretion of the attending senior anesthesiologists and cardiologists in the ICU. Patients with and without diabetes with persistently elevated serum glucose $(>180 \mathrm{mg} / \mathrm{dL})$ received IV insulin infusions to maintain serum glucose $<180 \mathrm{mg} / \mathrm{dL}$ for the duration of ICU care. The serum levels of potassium and other electrolytes were monitored and maintained within the normal range. An unpaired $t$ test and Fisher's exact test were used for comparison of between-group differences in mean values and categorical data, respectively. This study was approved by the ethics committee of Nippon Medical School. The committee has established that retrospective studies involving analysis of medical records can be conducted without informed consent from the subjects.

\section{Results}

Clinical characteristics and perioperative data are shown in Table 1 . There were no statistically significant differences between the landiolol and control groups in age, New York Heart Association (NYHA) class, history of hypertension, type of surgery, ejection fraction or preoperative $\beta$-blocker use. Overall, 17 of 105 patients (16.2\%), 5 of 57 (8.8\%) in the landiolol group and 12 of 48 (25\%) in the control group, developed postoperative AF $(P=0.03)$. In the landiolol group, AF developed in 2 patients on postoperative day 2 , in 1 patient on postoperative day 3 , and in 2 patients on postoperative day 4. The mean duration of landiolol administration was $23.9 \pm 32.0 \mathrm{~h}$. In addition, the time of AF onset after admission to the ICU was $3.0 \pm 1.2$ days in the intraoperative landiolol group, and $2.2 \pm 1.0$ days in the group that did not receive landiolol. Although the difference was not significant, onset tended to be delayed in the intraoperative landiolol group. Postoperative $\beta$-blockers were more frequently used in the landiolol group than in the control group $(\mathrm{P}<0.0001)$. The patients in the landiolol group showed a trend toward shorter times to extubation and length of ICU stay, but the differences between the groups were not statistically significant. There were no differences between the groups in blood glucose or potassium levels, and there were no significant differences in postoperative complications. 
Table 1 Clinical characteristics and perioperative data for the landiolol and control groups

\begin{tabular}{|c|c|c|c|}
\hline & Control & Landiolol & $P$ value \\
\hline & $(n=48)$ & $(n=57)$ & \\
\hline Age (yr) & $65.7 \pm 12.6$ & $67.2 \pm 11.5$ & NS \\
\hline NYHA class & $1.9 \pm 0.7$ & $1.9 \pm 0.7$ & NS \\
\hline BMI & $23.0 \pm 4.1$ & $23.0 \pm 3.4$ & NS \\
\hline Hypertension & $38(79.2)$ & $47(82.4)$ & NS \\
\hline Diabetes & $14(29.2)$ & $18(31.6)$ & NS \\
\hline Hyperlipidemia & $18(37.5)$ & $25(43.9)$ & NS \\
\hline Previous CHF & $22(45.8)$ & $23(40.4)$ & NS \\
\hline Previous Ml & $7(14.6)$ & $12(21.1)$ & NS \\
\hline Previous AF & $8(16.7)$ & $10(17.5)$ & NS \\
\hline LV Ejection fraction (\%) & $60.5 \pm 14.7$ & $56.4 \pm 15.2$ & NS \\
\hline Preoperative ACE inhibitor use & $38(79.2)$ & $37(64.9)$ & NS \\
\hline Preoperative $\beta$-blocker use & $24(50.0)$ & $29(50.9)$ & NS \\
\hline Postoperative $\beta$-blocker use & $16(33.3)$ & $41(71.9)$ & $<0.0001$ \\
\hline Preoperative $\mathrm{Cr}$ (mg/dL) & $1.1 \pm 1.8$ & $1.3 \pm 1.6$ & NS \\
\hline Preoperative \% VC & $95 \pm 21$ & $94 \pm 19$ & NS \\
\hline Preoperative FEV 1.0\% & $82 \pm 15$ & $82 \pm 14$ & NS \\
\hline On-pump CABG & $5(10.4)$ & $9(15.8)$ & NS \\
\hline Off-pump CABG & $14(29.2)$ & $27(47.4)$ & NS \\
\hline \multicolumn{4}{|l|}{ Valve surgery } \\
\hline AVR & $16(33.3)$ & $12(21.1)$ & NS \\
\hline MVR & $3(6.3)$ & $2(3,5)$ & NS \\
\hline MVP & $9(18.8)$ & $6(10.5)$ & NS \\
\hline TVP & $1(2.1)$ & $1(1.8)$ & NS \\
\hline Operating time (min) & $344 \pm 78$ & $334 \pm 86$ & NS \\
\hline Blood loss (mL) & $1159 \pm 1036$ & $1197 \pm 1246$ & NS \\
\hline Fluid and blood infusion volume (mL) & $5471 \pm 1657$ & $5405 \pm 1948$ & NS \\
\hline Urine volume $(\mathrm{mL})$ & $1283 \pm 933$ & $1270 \pm 948$ & NS \\
\hline No. of patients receiving intraoperative remifentanil & $32(66.7)$ & $39(68.4)$ & NS \\
\hline Intraoperative remifentanil dose ( $\mu \mathrm{g} / \mathrm{kg} / \mathrm{min})$ & $0.18 \pm 0.06$ & $0.21 \pm 0.07$ & NS \\
\hline Postoperative AF & $12(25.0)$ & $5(8.8)$ & 0.02 \\
\hline Time of onset of new AF (d) & $2.2 \pm 1.0$ & $3.0 \pm 1.2$ & NS \\
\hline Length of ICU stay (d) & $3.5 \pm 2.0$ & $3.0 \pm 2.1$ & NS \\
\hline Time to extubation (hr) & $20.9 \pm 41.6$ & $16.0 \pm 29.1$ & NS \\
\hline \multicolumn{4}{|l|}{ Postoperative complications } \\
\hline Pulmonary edema & $6(12.5)$ & $7(12.3)$ & NS \\
\hline Stroke & $1((2.1)$ & 0 & NS \\
\hline Seizures & $1(2.1)$ & $1(1.8)$ & NS \\
\hline Renal failure & 0 & $1(1.8)$ & NS \\
\hline
\end{tabular}

Values are shown as numbers (\%) or as the mean \pm SD. The unpaired $t$-test was used for comparison of between-group differences in means. Fisher's exact test was used for comparison of between-group differences in categorical data. NYHA: New York Heart Association, CHF: congestive heart failure, MI: myocardial infarction, $L V$ : left ventricular, ACE: angiotensin-converting enzyme, $C r$ : creatinine, AF: atrial fibrillation, ICU: intensive care unit. 
Table 2 Systolic and diastolic blood pressure and heart rate in the two groups

\begin{tabular}{lllllll}
\hline & Group & Before induction & After induction & Start of operation & Sternotomy & End of operation \\
\hline SBP $(\mathrm{mm} \mathrm{Hg})$ & Control & $136 \pm 18$ & $88 \pm 12$ & $115 \pm 19$ & $128 \pm 15$ & $115 \pm 19$ \\
& Landiolol & $139 \pm 24$ & $93 \pm 13$ & $120 \pm 19$ & $137 \pm 18^{*}$ & $118 \pm 19$ \\
DBP (mm Hg) & Control & $69 \pm 16$ & $44 \pm 7$ & $56 \pm 11$ & $63 \pm 12$ & $54 \pm 9$ \\
& Landiolol & $72 \pm 17$ & $46 \pm 10$ & $58 \pm 14$ & $65 \pm 15$ & $56 \pm 11$ \\
HR (beats/min) & Control & $68 \pm 12$ & $54 \pm 10$ & $59 \pm 11$ & $65 \pm 8$ & $76 \pm 9$ \\
& Landiolol & $70 \pm 12$ & $58 \pm 13$ & $63 \pm 11^{*}$ & $71 \pm 12^{*}$ & $75 \pm 15$ \\
\hline
\end{tabular}

Values are shown as the mean $\pm \mathrm{SD}$. ${ }^{*} \mathrm{P}<0.05$ vs. control (unpaired $t$-test). SBP: systolic blood pressure, DBP: diastolic blood pressure, $H R$ : heart rate. Repeated measures ANOVA showed significant differences between the groups for SBP $(p=0.03)$ and HR $(p=0.02)$. $S B P$ was significantly higher in the landiolol group at the start of the operation. SBP and $H R$ were significantly higher in the landiolol group at sternotomy. $D B P$ was similar in both groups.

Intraoperative systolic blood pressure (SBP), diastolic blood pressure (DBP), and HR are shown in Table 2. In the landiolol group SBP was significantly higher at the start of the operation and SBP and HR were significantly higher at sternotomy, compared to the control group. The incidences of AF after valve surgery and off-pump coronary artery bypass grafting (CABG) were lower in the landiolol group (Table 3), but the differences between the groups were not statistically significant. The percentage incidence of postoperative AF was similar among all operative procedures (Table 3 ).

The data in Table 4 suggest a lower incidence of postoperative AF in patients who received postoperative $\beta$-blocker therapy $(12.3 \%, 7$ of 57 patients) compared with those who did not receive this therapy (20.8\%, 10 of 48 patients). However, this difference was not statistically significant. Of 57 patients in the landiolol group, $\beta$-blocker therapy (landiolol, carvedilol or atenolol) was continued postoperatively in 41 and discontinued after surgery in 16. There was no significant difference in the occurrence of postoperative AF between these subgroups (9.8\% vs. 6.3\%, respectively). Patients who received $\beta$-blocker therapy only after surgery had an incidence of postoperative AF of $18.8 \%$ (3 of 16). A higher rate of AF was found in patients who did not receive $\beta$-blockers perioperatively (28.1\%, 9 of 32), but the occurrence of postoperative AF did not differ significantly among all the sub-groups.

Table 3 Comparison of the incidence of postoperative atrial fibrillation between the landiolol and control groups

\begin{tabular}{lllll}
\hline & Total population & Control & Landiolol & P value \\
\hline Valve surgery & $9 / 50(18 \%)$ & $7 / 29(24 \%)$ & $2 / 21(10 \%)$ & 0.18 \\
On-pump CABG & $2 / 14(14 \%)$ & $1 / 5(20 \%)$ & $1 / 9(11 \%)$ & 0.65 \\
Off-pump CABG & $6 / 41(15 \%)$ & $4 / 14(29 \%)$ & $2 / 27(7 \%)$ & 0.07 \\
All surgery & $17 / 105(16 \%)$ & $12 / 48(25 \%)$ & $5 / 57(8.8 \%)$ & 0.02 \\
\hline
\end{tabular}

Fisher's exact test was used to analyze between-group differences in categorical data. CABG: coronary artery bypass grafting.

\section{Discussion}

AF is a common complication after cardiovascular surgery and often results in prolonged hospital stays and increased morbidity compared with patients who maintain sinus rhythm [2-5]. Approaches to preoperative and postoperative prophylaxis of AF using selective $\beta$-blockers $[1,8,9]$, amiodarone [6], and atrial pacing [7] have achieved varying degrees of clinical success. However, the role of intraoperative $\beta$-adrenergic blockers in preventing postoperative AF is unknown. In the present study, patients who received intraoperative landiolol had a significantly lower incidence of postoperative AF (8.8\%).

In a study of patients who underwent on-pump CABG, an inhibitory effect on postoperative AF was reported from administering landiolol for $48 \mathrm{~h}$ from the start of surgery [16]. The results of our study, which includes not only on-pump CABG, but also off-pump CABG and heart valve surgical patients, suggest that AF occurrences can be suppressed even in these cases. Sezai et al. [16] reported that postoperative AF was reduced by treatment with landiolol in patients undergoing CABG on cardiopulmonary bypass (10\%, 7 of 70 patients). Landiolol infusion was started at the time of central anastomosis during CABG and discontinued after $48 \mathrm{~h}$. In our study, of 57 patients in the landiolol group, $\beta$-blocker therapy was continued postoperatively in 41 patients and discontinued after surgery in 16. The incidence of postoperative AF in patients receiving intraoperative and postoperative $\beta$-blocker

Table 4 Effect of perioperative $\boldsymbol{\beta}$-blockers on the incidence of postoperative AF

\begin{tabular}{lll}
\hline $\begin{array}{l}\text { Intraoperative } \\
\beta \text {-blocker use }\end{array}$ & $\begin{array}{l}\text { Postoperative } \\
\beta \text {-blocker use }\end{array}$ & $\begin{array}{l}\text { Incidence of } \\
\text { postoperative AF }\end{array}$ \\
\hline$(+)$ & $(+)$ & $4 / 41(9.8 \%)$ \\
$(+)$ & $(-)$ & $1 / 16(6.3 \%)$ \\
$(-)$ & $(+)$ & $3 / 16(18.8 \%)$ \\
$(-)$ & $(-)$ & $9 / 32(28.1 \%)$ \\
\hline
\end{tabular}

AF: atrial fibrillation. There were no significant differences in the incidence of postoperative AF among the groups. Four of the 41 patients who received intraoperative and postoperative $\beta$-blockers developed postoperative AF. Two of these patients developed AF after discontinuation of postoperative $\beta$-blocker therapy. 
therapy was $9.8 \%$, which was similar to the values in their study. Similarly, the incidence of postoperative AF in patients who received landiolol only during surgery was 6.3\%. Most known risk factors were found to be similar among groups. These results suggest a possible role for prophylactic landiolol during cardiac surgery to reduce the incidence of postoperative AF.

In the postoperative heart, multiple factors may potentially predispose a patient to AF through alterations in refractoriness and/or local reentry. These include operative trauma from surgical dissection and manipulation, inflammation, elevation in atrial pressure from postoperative impaired ventricular function, chemical stimulation during perioperative support with catecholamines and other inotropic agents, reflex sympathetic activation from volume loss, anemia or pain, parasympathetic activation, fever from atelectasis or infection, and hypoglycemia or ischemic damage incurred during surgery $[17,18]$. Recent studies have shown that markers of oxidative injury and inflammation, such as C-reactive protein, are elevated in patients with AF, indicating that they may have an important role in the pathogenesis of postoperative AF $[19,20]$. Landiolol has a lipid peroxidation-reducing effect and suppresses the increase in phospholamban serine phosphorylation in the sarcoplasmic reticulum (SR) in hearts subjected to ischemiareperfusion (I/R) [21]. Landiolol, as well as propranolol and esmolol, also has cardioprotective effects in isolated guinea pig hearts subjected to I/R injury [22]. Therefore, landiolol may have anti-ischemic properties as an antioxidant and via preservation of SR function during the ischemic period. In addition, preischemic administration of landiolol reduced cardiac cellular damage and improved the recovery of cardiac function in the rat heart through protein kinase $\mathrm{C}$ (PKC) epsilon-mediated pathway, similar to that afforded by ischemic preconditioning [23]. Reduction of postoperative AF by landiolol may be due to reduced inflammation or oxidative stress, or pharmacological preconditioning effect, in addition to effective $\beta$-blockade.

Cardiac surgery requiring cardiopulmonary bypass results in significant impairment of $\beta$-adrenergic receptor $(\beta A R)$ function [24]. This can cause depressed postoperative myocardial function that results in atrial enlargement, which is a risk for postoperative AF. The possible mechanisms underlying impaired $\beta A R$ function include myocardial ischemia-reperfusion injury [25], a hibernating and stunned myocardium [26], and acute myocardial $\beta A R$ desensitization $[27,28]$. A marked increase in catecholamine levels found during cardiac surgery resulting in agonist-induced desensitization may be a major mechanism behind acute $\beta A R$ desensitization [27]. Increased myocardial catecholamine concentrations may, at least in part, be the stimulus for the myocardial hyporesponsiveness seen during cardiac surgery. In support of this, blockade of $\beta$ ARs with low-dose $\beta A R$ antagonist therapy improves myocardial function in congestive heart failure [29], a clinical setting in which attenuation of chronic $\beta A R$ desensitization has been postulated as a possible mechanism. In the landiolol group SBP was significantly higher at the start of the operation and SBP and HR were significantly higher at sternotomy, compared to the control group. These results suggested that serum catecholamine levels in the landiolol group may increase during surgery. Nevertheless, we found a marked reduction of postoperative AF in the landiolol group. Landiolol may have the suppressive effect of catecholamine-induced $\beta A R$ desensitization. Booth et al. reported that intravenous esmolol administration during coronary artery revascularization after acute myocardial ischemia results in improved left ventricular function immediately after cardiopulmonary bypass $(\mathrm{CPB})$ via enhanced $\beta A R$ signaling [30], whereas chronic oral $B$-adrenergic blocker therapy in patients undergoing CABG surgery does not protect patients from acute $\beta A R$ desensitization [27]. In addition, Cork et al. demonstrated improved early intermediate outcomes (such as cardiac output) immediately post-CPB in patients receiving an intraoperative $\beta$-adrenergic blocker [31]. In a similar manner, improvement of $\beta A R$ function by intraoperative administration of landiolol may contribute to preserved left ventricular function and fewer inotropic requirements postoperatively, resulting in a reduced incidence of postoperative AF.

The prevalence of AF increases with worsening heart failure from $5 \%$ in patients of NYHA class I under drug therapy to as high as $50 \%$ in those of NYHA class IV. Structural, electrophysiologic, and neurohumoral mechanisms may play important roles in this effect, but an association of heart failure and AF has not been established in the postoperative setting. Factors predisposing patients to postoperative AF may be unrelated to left ventricular function, and in our study patients who received landiolol intraoperatively experienced less AF than those who did not, despite having similar left ventricular ejection fractions (56.4\% vs. $60.5 \%$ ). However, preoperative left ventricular dysfunction is one of the strongest predictors of mortality after CABG surgery. The use of ultrashort-acting $\beta$-blockers has been suggested as a safe and efficacious method for treating patients with accelerated adrenergic drive during and after the postoperative phase of cardiovascular surgery. Landiolol hydrochloride is approximately nine times more potent in $\beta$-blocking activity in vivo and eight times more cardioselective in vitro than esmolol [10]. Moreover, the suppressive effects on cardiovascular perforance are significantoly less potent or landiolol than those of esmolol at equipotent $\beta$-blocking doses [13]. These properties facilitate a more rapid recovery after drug administration if an adverse effect develops, 
which should lead to safer perioperative management of patients undergoing cardiac surgery and in other clinical settings.

Our study had several linitations. First, our patients had undergone various cardiac procedures with or without the use of a heart-lung machine. Second, they were not randomly assigned to either group and there was a between-group difference in the number and type of the cardiac surgery that the patients had undergone. Third, the optimum dose for intraoperative landiolol could not be verified, including in our investigations. Therefore, to confirm the preventive effect of intraoperative landiolol on AF after cardiac surgery, a large-scale clinical study is needed.

The main points of our findings are: (1) when landiolol was not administered intraoperatively, the incidence of postoperative AF was lower in the group that received postoperative $\beta$-blockers than in the group that did not; (2) even when postoperative $\beta$-blockers were used, the incidence of postoperative AF was lower in the group that received intraoperative landiolol; and (3) when landiolol was administered intraoperatively, the incidence of postoperative AF was low regardless of whether or not postoperative $\beta$-blockers were used.

\section{Conclusions}

Although this was a retrospective, nonrandomized study, our retrospective review of 105 patients who underwent cardiac surgery demonstrated a marked reduction in postoperative AF (8.8\%) in those who received landiolol during surgery. In the future we expect to conduct a randomized prospective study to investigate the inhibitory effects of intraoperative landiolol on postoperative $\mathrm{AF}$, in patients who have undergone off-pump CABG and heart valve surgery.

\section{Abbreviations}

AF: Atrial fibrillation; $\beta A R$ : $\beta$-adrenergic receptor; CABG: Coronary artery bypass grafting; CPB: Cardiopulmonary bypass; DBP: Diastolic blood pressure; HR: Heart rate; ICU: Intensive care unit; NYHA: New York Heart Association, SBP: Systolic blood pressure.

\section{Competing interests}

The authors declare that they have no competing interests.

\section{Authors' contributions}

All authors have made substantial contribution to study conception and design, acquisition and analysis of data, and manuscript preparation, and have approved the final manuscript.

Received: 13 March 2012 Accepted: 11 January 2013

Published: 24 January 2013

\section{References}

1. Mitchell LB: Incidence, timing and outcome of atrial tachyarrhythmias after cardiac surgery. In Atrial Fibrillation after Cardiac Surgery. Edited by Steinberg JS. Boston: Kluwer Academic Publishers; 2000:37-50

2. Hogue CW Jr, Creswell LL, Gutterman DD, Fleisher LA, American College of Chest Physicians: Epidemiology, mechanisms, and risks: American college of chest physicians guidelines for the prevention and management of postoperative atrial fibrillation after cardiac surgery. Chest 2005, 128:9S-16S.

3. Mathew JP, Parks R, Savino JS, Friedman AS, Koch C, Mangano DT, Browner WS: Atrial fibrillation following coronary artery bypass surgery: predictors, outcomes, and resource utilization. JAMA 1996, 276:300-306.

4. Creswell LL, Schuessler RB, Rosenbloom M, Cox JL: Hazards of postoperative atrial arrhythmias. Ann Thorac Surg 1993, 56:539-549.

5. Aranki SF, Shaw DP, Adams DH, Rizzo RJ, Couper GS, VanderVliet M, Collins $J J \mathrm{Jr}$, Cohn LH, Burstin HR: Predictors of atrial fibrillation after coronary artery surgery: current trends and impact on hospital resources. Circulation 1996, 94:390-397.

6. Mitchell LB, Exner DV, Wyse DG, Connolly CJ, Prystai GD, Bayes AJ, Kidd WT, Kieser T, Burgess JJ, Ferland A, MacAdams CL, Maitland A: Prophylactic oral amiodarone for the prevention of arrhythmias that begin early after revascularization, valve replacement, or repair: PAPABEAR: a randomized controlled trial. JAMA 2005, 294:3093-3100.

7. Maisel WH, Epstein AE, American College of Chest Physicians: The role of cardiac pacing: American college of chest physicians guidelines for the prevention and management of postoperative atrial fibrillation after cardiac surgery. Chest 2005, 128:36S-38S.

8. Bradley D, Creswell LL, Hogue CW Jr, Epstein AE, Prystowsky EN, Daoud EG, American College of Chest Physicians: Pharmacologic prophylaxis: American college of chest physicians guidelines for the prevention and management of postoperative atrial fibrillation after cardiac surgery. Chest 2005, 128:395-475.

9. DiDomenico RJ, Massad MG: Pharmacologic strategies for prevention of atrial fibrillation after open heart surgery. Ann Thorac Surg 2005, 79:728-740.

10. Iguchi S, Iwamura H, Nishizaki M, Hayashi A, Senokuchi K, Kobayashi K, Sakaki K, Hachiya K, Ichioka Y, Kawamura M: Development of a highly cardioselective ultra short-acting [beta]-blocker, ONO-1101. Chem Pharm Bull 1992, 40:1462-1469.

11. Motomura S, Hagihara A, Narumi Y, Hashimoto K: Time course of a new ultrashort-acting beta-adrenoceptor-blocking drug, ONO-1101: comparison with those of esmolol and propranolol by using the canine isolated, blood-perfused heart preparations. I Cardiovasc Pharmacol 1998, 31:431-440

12. Muraki K, Nakagawa $\mathrm{H}$, Nagano $\mathrm{N}$, Henmi $\mathrm{S}$, Kawasumi H, Nakanishi T, Imaizumi K, Tokuno T, Atsuki K, Imaizumi Y, Watanabe M: Effects of ONO1101, a novel beta-antagonist, on action potential and membrane currents in cardiac muscle. J Pharmacol Exp Ther 1996, 278:555-563.

13. Sugiyama A, Takahara A, Hashimoto K: Electrophysiologic cardiohemodynamic and beta-blocking actions of a new ultrashortacting beta-blocker, ONO-1101, assessed by the in vivo canine model in comparison with esmolol. J Cardiovasc Pharmacol 1999, 34:70-77.

14. Takahashi S, Fujii Y, Inomata S, Miyabe M, Toyooka H: Landiolol decreases a dysrhythmogenic dose of epinephrine in dogs during halothane anesthesia. Can J Anaesth 1999, 46:599-604.

15. Atarashi H, Kuruma A, Yashima M, Saitoh H, Ino T, Endoh Y, Hayakawa H: Pharmacokinetics of landiolol hydrochloride, a new ultra-short-acting beta-blocker, in patients with cardiac arrhythmias. Clin Pharmacol Ther 2000, 68:143-150.

16. Sezai A, Minami K, Nakai T, Hata M, Yoshitake I, Wakui S, Shiono M, Hirayama A: Landiolol hydrochloride for prevention of atrial fibrillation after coronary artery bypass grafting: new evidence from the PASCAL trial. J Thoracic Cardiovasc Surg 2011, 141:1478-1487.

17. Caretta Q, Mercanti CA, De Nardo D, Chiarotti F, Scibilia G, Reale A, Marino B: Ventricular conduction defects and atrial fibrillation after coronary artery bypass grafting: multivariate analysis of preoperative, intraoperative and postoperative variables. Eur Heart J 1991, 12:1107-1111.

18. Crosby LH, Pifalo WB, Woll KR, Burkholder JA: Risk factors for atrial fibrillation after coronary artery bypass grafting. Am J Cardiol 1990, 66:1520-1522

19. Ishida K, Kimura F, Imamaki M, Ishida A, Shimura H, Kohno H, Sakurai M, Miyazaki M: Relation of inflammatory cytokines to atrial fibrillation after off-pump coronary artery bypass grafting. Eur J Cardiothorac Surg 2006, 29:501-505

20. Chung MK, Martin DO, Sprecher D, Wazni O, Kanderian A, Carnes CA, Bauer JA, Tchou PJ, Niebauer MJ, Natale A, Van Wagoner DR: C-reactive protein elevation in patients with atrial arrhythmias: inflammatory mechanisms and persistence of atrial fibrillation. Circulation 2001, 104:2886-2891. 
21. Kimura-Kurosawa S, Kanaya N, Kamada N, Hirata N, Nakayama M, Namiki A: Cardioprotective effect and mechanism of action of landiolol on the ischemic reperfused heart. J Anesth 2007, 21:480-489.

22. Kurosawa S, Kanaya N, Niiyama Y, Nakayama M, Fujita S, Namiki A: Landiolol, esmolol and propranolol protect from ischemia/reperfusion injury in isolated guinea pig hearts. Can J Anaesth 2003, 50:489-494.

23. Takahashi Y, Takemura S, Minamiyama Y, Shibata T, Hirai H, Sasaki Y, Sakaguchi M, Suehiro S: Landiolol has cardioprotective effects against reperfusion injury in the rat heart via the PKCepsilon signaling pathway. Free Radic Res 2007, 41:757-769.

24. Schwinn DA, Leone BJ, Spahn DR, Chesnut LC, Page SO, McRae RL, Liggett SB: Desensitization of myocardial beta-adrenergic receptors during cardiopulmonary bypass. Evidence for early uncoupling and late downregulation. Circulation 1991, 84:2559-2567.

25. Toleikis PM, Tomlinson CW: Myocardial functional preservation during ischemia: influence of beta blocking agents. Mol Cell Biochem 1997, 176:205-210.

26. Shattock MJ: Myocardial stunning: do we know the mechanism? Basic Res Cardiol 1997, 92:18-22.

27. Booth JV, Landolfo KP, Chesnut LC, Bennett-Guerrero E, Gerhardt MA, Atwell DM, El-Moalem HE, Smith MS, Funk BL, Kuhn CM, Kwatra MM, Schwinn DA: Acute depression of myocardial beta-adrenergic receptor signaling during cardiopulmonary bypass: impairment of the adenylyl cyclase moiety. Duke Heart Center Perioperative Desensitization Group. Anesthesiology 1998, 89:602-611.

28. Gerhardt MA, Booth JV, Chesnut LC, Funk BL, El-Moalem H, Kwatra MM, Schwinn DA: Acute myocardial beta-adrenergic receptor dysfunction after cardiopulmonary bypass in patients with cardiac valve disease. Duke Heart Center Perioperative Desensitization Group. Circulation 1998, 98:||275-1||281.

29. Bristow MR: What type of beta-blocker should be used to treat chronic heart failure? Circulation 2000, 102:484-486.

30. Booth JV, Spahn DR, McRae RL, Chesnut LC, El-Moalem H, Atwell DM, Leone BJ, Schwinn DA: Esmolol improves left ventricular function via enhanced beta-adrenergic receptor signaling in a canine model of coronary revascularization. Anesthesiology 2002, 97:162-169.

31. Cork RC, Azari DM, McQueen KA, Aufderheide S, Mitchell M, Naraghi M: Effect of esmolol given during cardiopulmonary bypass on fractional area of contraction from transesophageal echocardiography. Anesth Analg 1995, 81:219-224.

doi:10.1186/1749-8090-8-19

Cite this article as: Nakanishi et al:: Postoperative atrial fibrillation in patients undergoing coronary artery bypass grafting or cardiac valve surgery: intraoperative use of landiolol. Journal of Cardiothoracic Surgery 2013 8:19.

\section{Submit your next manuscript to BioMed Central and take full advantage of:}

- Convenient online submission

- Thorough peer review

- No space constraints or color figure charges

- Immediate publication on acceptance

- Inclusion in PubMed, CAS, Scopus and Google Scholar

- Research which is freely available for redistribution 